\title{
Effects of Comorbid Factors on Prognosis of Three Different Geriatric Groups with COVID-19 Diagnosis
}

\author{
Özkan Görgülü ${ }^{1}$ (D) Murat Duyan ${ }^{2}$ (D) \\ Accepted: 13 November 2020 / Published online: 18 November 2020 \\ (C) Springer Nature Switzerland AG 2020
}

\begin{abstract}
Coronavirus disease 2019 (COVID-19) is a new zoonotic infectious disease that was first reported to the World Health Organization (WHO) on December 31, 2019, and declared as a pandemic by WHO on March 11, 2020. Due to the increased incidence of multimorbidity in geriatric age groups, COVID-19 disease leads to more severe consequences in the elderly. We aimed to determine the effects of age, comorbidity factors, symptoms, laboratory findings, and radiological results on prognosis by dividing our patients into 3 different geriatric age groups, using a retrospective descriptive analysis method. Patients included in the retrospective study $(n=483)$ were divided into the following three different geriatric age groups: young-old (65-74 years), middle-aged (75-84 years), and the oldest-elderly ( 85 years and over).The length of stay in the intensive care unit of the patients between the ages of 75-84 was higher than the other two groups $(p=0.013)$. Mortality rates were lowest in patients aged 6574 years $(p<0.001)$. The rate of ground glass opacity in thorax CT was higher in patients with mortality $(p<0.001)$. While the rate of COPD-bronchial asthma was higher in surviving patients $(p=0.001)$, malignancy $(p=0.005)$ and cerebrovascular disease $(p<0.001)$ were higher in patients who died. Patients aged between 75 and 84 (OR: $2.602 ; 95 \%$ CI: $1.306-5.183 ; p=0.007)$ or $\geq$ 85 (OR: 4.086 ; 95\% CI: $1.687-9.9 ; p=0.002$ ) had higher risk for mortality compared to patients aged between 65 and 74 . The lowest mortality rates were observed in patients aged 65-74 years. Among the supportive diagnostic methods in 3 different geriatric age groups, PCR positivity has no effect on mortality, while the ground glass opacity on tomography is closely related to the need for intensive care and increased mortality. In patients with COPD-bronchial asthma comorbidity and those with symptoms of fatigue, dry cough, and sore throat, transfer to intensive care and mortality rates were lower, while patients who were transferred to intensive care and who developed mortality had higher malignancy and cerebrovascular disease comorbidities and dyspnea symptoms.
\end{abstract}

Keywords COVID-19 · SARS-CoV-2 · Coronavirus · Intensive care units · Geriatrics · Real-time polymerase chain reaction · Tomography $\cdot$ Death $\cdot$ Prognosis

This article is part of the Topical Collection on COVID-19

Özkan Görgülü

drozkangorgulu@hotmail.com

Murat Duyan

drmuratduyan@gmail.com

1 Department of Anesthesiology and Reanimation, Antalya Training and Research Hospital, Varlık Mh. Kazım Karabekir Cd, 07100 Antalya, Turkey

2 Department of Emergency Medicine, Antalya Training and Research Hospital, Antalya, Turkey

\section{Introduction}

Coronavirus disease 2019 (COVID-19) is a new zoonotic infectious disease that was first reported to the World Health Organization (WHO) on December 31, 2019, and was declared as a pandemic by WHO on March 11, 2020 [1]. COVID-19 continues to disproportionately affect older adults, with severe patient losses, from hospitalization to increased risk of death, causing severe illness [2]. The COVID-19 pandemic has affected millions of people in more than 180 regions, and patients diagnosed with cancer, as well as older adults, appear to be particularly vulnerable to the morbidity and mortality associated with COVID-19, meaning that older adults with cancer constitute a particularly high-risk population [3]. Elderly patients with head and neck cancer can be 
expected to have significantly higher COVID-19 severity and mortality [4]. Although most people infected with SARSCoV-2 have the potential of recovery, mortality in those who develop severe disease increases with the presence of multimorbidity and with comorbidity in the elderly $[5,6]$. Higher rates of morbidity and mortality in the elderly have been associated with accompanying comorbidities, particularly cardiovascular disease, and weakness and physiological weakness that weaken the immune response [6]. Vitamin D deficiency is more common in the elderly population, and this considerably increases mortality [7]. Advances in various diagnostic approaches such as real-time polymerase chain reaction (rRT-PCR), chest radiography, and computed tomography (CT) imaging and other modern diagnostic methods for this infection have been emphasized [8]. In line with this information, we planned this study to determine the important effects of age, comorbidity factors, symptoms, laboratory findings, and radiological results on prognosis of patients with COVID-19 symptoms in 3 different geriatric age groups.

\section{Materials and Methods}

\section{Patient Population}

This study was conducted in accordance with the Declaration of Helsinki from March 2020 to June 2020 after obtaining approval from the Institutional Ethics Committee (Date of Approval-Protocol No: 03.06.2020-7/8).

This study was conducted as a single-center retrospective descriptive study by scanning the electronic health record system (SARUS) data and patient files of 483 geriatric patients aged 65 and over with COVID-19 symptoms among 3628 patients who applied to our level 3 hospital with COVID-19 symptoms. Among this group, 483 patients aged 65 and over, $278(57.6 \%)$ consisting of women, were included into the study. Three thousand one hundred forty-five patients were excluded because they were under 65 years old. During the study period, patients aged 65 years and over who were admitted to our hospital but did not have COVID-19 symptoms were not included in the study.

\section{Assessment}

Patients included in the study $(n=483)$ were grouped into the following three different geriatric age groups: young-old (6574 years), middle-old ( $75-84$ years), and oldest-old ( 85 years and above). Among the sample patient group, $7.7 \%$ (280) were $65-74,3.7 \%$ (133) were $75-84$, and $1.9 \%$ (70) were 85 and over. In the patient group, we identified fever, fatigue, dry cough, sore throat, shortness of breath, chest pain, headache, dizziness, diarrhea, and nausea-vomiting as symptoms of COVID-19. Hypertension (HT), diabetes mellitus (DM), chronic obstructive pulmonary disease (COPD), bronchial asthma, malignancy, cardiovascular disease, cerebrovascular disease, chronic kidney failure, chronic liver disease, heart failure, and hyperlipidemia were evaluated.

Analysis of comorbidity factors, symptoms, laboratory findings and radiological findings, and their effect on prognosis were evaluated in 3 different geriatric age groups with COVID-19 symptoms.

\section{Statistical Analysis}

Statistical analysis was made using IBM SPSS Statistics for Windows, Version 23.0 (IBM Corp., Armonk, NY). The normality assumptions were controlled by the Shapiro-Wilk test. Descriptive analyses were presented using mean \pm SD, median (range), or $n(\%)$, where appropriate. Categorical data were analyzed by Pearson chi-square and Fisher's exact test. Mann-Whitney $U$ test and Student's $t$ test were used for analysis of non-normally and normally distributed numerical data, respectively. The Kruskal-Wallis test was used for comparison of non-parametric variables between groups, and the Bonferroni-Dunn test was used as a post hoc test for significant cases, while one-way ANOVA with post hoc Tukey HSD test was used for parametric variables. The receiver operating characteristic (ROC) curve analysis was applied to determine optimal cutoff point of age and CRP for mortality, and area under the curve (AUC), sensitivity, and specificity were calculated and reported with $95 \%$ confidence intervals. Multivariate logistic regression analysis was used to determine independent risk factors associated with mortality. Odds ratio (OR) with corresponding $95 \%$ confidence intervals (95\% CIs) was reported. A $p$ value of less than 0.05 was considered statistically significant.

\section{Results}

In a total of 3628 COVID-19 symptomatic patients, $13.3 \%$ (483) were in the geriatric group, $7.7 \%$ (280) of them were $65-74,3.7 \%$ (133) were $75-84$, and $1.9 \%$ (70) were 85 and over.

The average age of 483 patients with COVID-19 symptoms was $74.4 \pm 7.94 .42 .4 \%$ (205) of the patients were male and $57.6 \%$ (278) were female. There was no significant difference in gender distribution by age groups $(p=0.366)$.

For diabetes mellitus ( $p=0.250$ ), COPD-bronchial asthma $(p=0.572)$, malignancy $(p=0.101)$, chronic renal failure $(p=$ $0.482)$, chronic liver disease $(p=0.083)$, heart failure $(p=$ 0.288 ), and hyperlipidemia $(p=0.850)$, no significant difference was observed in terms of their incidence rates.

The incidence of hypertension was higher in patients aged $75-84(84.2 \%)$ compared to patients aged $65-74$ years $(61.1 \%)$ and 85 and over $(68.6 \%)(p<0.001)$. 
The incidence of cardiovascular disease was higher in patients aged 75-84 (47.4\%) compared to patients aged 65-74 (34.6\%) $(p=0.044)$.

The incidence of cerebrovascular disease in patients aged 75-84 years $(29.3 \%)$ was higher than patients aged $65-$ 74 years $(14.6 \%)$ and 85 years of age $(15.7 \%)(p<0.001)$.

Symptoms of fever $(p=0.021)$, weakness $(p=0.001)$, sore throat $(p<0.001)$, dizziness $(p=0.001)$, and myalgia $(p<0.001)$ were higher in patients aged 85 years and older.

While the frequency of dyspnea was lower in patients aged 65-74 years compared to other age groups $(p=0.020)$, the rate of chest pain was higher $(p=0.020)$.

It was determined that there was no significant difference between the age groups in terms of the incidence of dry cough $(p=0.186)$, headache $(p=0.190)$, diarrhea $(p=0.154)$, nauseavomiting $(p=0.974)$, and no smell-taste $(p=0.583)$ (Table 1$)$.
Although the rate of non-invasive mechanical ventilation was higher in patients aged $75-84$ years, this difference was not statistically significant $(p=0.065)$.

The rate of using invasive mechanical ventilation was lower in patients aged 65-74 years compared to the other two age groups $(p<0.001)$.

The highest rate of patients discharged from the emergency department was observed in patients between the ages of $65-74$, while the rate of admission to intensive care was highest in patients aged 75-84 years and over 85 years $(p<0.001)$.

The rate of patients transferred from service to intensive care was similar in all age groups $(p=0.109)$.

There was no significant difference in terms of length of stay of the patients hospitalized in the ward according to age groups $(p=0.275)$.
Table 1 Demographic characteristics of the patients

\begin{tabular}{|c|c|c|c|c|c|}
\hline & Total $(n: 483)$ & $65-74(n: 280)$ & $75-84(n: 133)$ & $\begin{array}{l}85 \text { and over } \\
(n: 70)\end{array}$ & $p$ \\
\hline Prevalence ( $n: 3628)$ & $13.3 \%$ & $7.7 \%$ & $3.7 \%$ & $1.9 \%$ & - \\
\hline Age & $74.4 \pm 7.94$ & $68.65 \pm 2.85^{\mathrm{a}}$ & $79.32 \pm 2.76^{\mathrm{b}}$ & $88.29 \pm 2.6^{\mathrm{c}}$ & $<0.001$ \\
\hline \multicolumn{6}{|l|}{ Gender } \\
\hline Female & $205(42.4)$ & $112(40)$ & $63(47.4)$ & $30(42.9)$ & \multirow[t]{2}{*}{0.366} \\
\hline Male & $278(57.6)$ & $168(60)$ & $70(52.6)$ & $40(57.1)$ & \\
\hline \multicolumn{6}{|l|}{ The comorbidities } \\
\hline Hypertension & $331(68.5)$ & $171(61.1)^{\mathrm{a}}$ & $112(84.2)^{\mathrm{b}}$ & $48(68.6)^{\mathrm{a}}$ & $<0.001$ \\
\hline Diabetes mellitus & $149(30.8)$ & $83(29.6)$ & $48(36.1)$ & $18(25.7)$ & 0.250 \\
\hline COPD-asthma-bronchitis & $186(38.5)$ & $111(39.6)$ & $52(39.1)$ & $23(32.9)$ & 0.572 \\
\hline Malignancy & $75(15.5)$ & $49(17.5)$ & $21(15.8)$ & $5(7.1)$ & 0.101 \\
\hline Cardiovascular disease & $186(38.5)$ & $97(34.6)^{\mathrm{a}}$ & $63(47.4)^{\mathrm{b}}$ & $26(37.1)^{\mathrm{a} . \mathrm{b}}$ & 0.044 \\
\hline Cerebrovascular disease & $91(18.8)$ & $41(14.6)^{\mathrm{a}}$ & $39(29.3)^{b}$ & $11(15.7)^{\mathrm{a}}$ & 0.001 \\
\hline Chronic renal failure & $44(9.1)$ & $23(8.2)$ & $12(9)$ & $9(12.9)$ & 0.482 \\
\hline Chronic liver disease & $17(3.5)$ & $14(5)$ & $3(2.3)$ & $0(0)$ & 0.083 \\
\hline Heart failure & $63(13)$ & $31(11.1)$ & $22(16.5)$ & $10(14.3)$ & 0.288 \\
\hline Hyperlipidemia & $12(2.5)$ & $6(2.1)$ & $4(3)$ & $2(2.9)$ & 0.850 \\
\hline \multicolumn{6}{|l|}{ The symptoms } \\
\hline Fever & $235(48.7)$ & $122(43.6)^{\mathrm{a}}$ & $71(53.4)^{a . b}$ & $42(60)^{\mathrm{b}}$ & 0.021 \\
\hline Weakness & $232(48)$ & $133(47.5)^{\mathrm{a}}$ & $52(39.1)^{\mathrm{a}}$ & $47(67.1)^{\mathrm{b}}$ & 0.001 \\
\hline Dry cough & $234(48.4)$ & $136(48.6)$ & $58(43.6)$ & $40(57.1)$ & 0.186 \\
\hline Throat ache & $189(39.1)$ & $115(41.1)^{\mathrm{a}}$ & $34(25.6)^{\mathrm{b}}$ & $40(57.1)^{\mathrm{c}}$ & $<0.001$ \\
\hline Shortness of breath & $278(57.7)$ & $146(52.3)^{\mathrm{a}}$ & $87(65.4)^{\mathrm{b}}$ & $45(64.3)^{\mathrm{b}}$ & 0.020 \\
\hline Chest pain & $51(10.6)$ & $38(13.6)^{\mathrm{a}}$ & $11(8.3)^{a . b}$ & $2(2.9)^{\mathrm{b}}$ & 0.020 \\
\hline Headache & $61(12.6)$ & $39(13.9)$ & $11(8.3)$ & $11(15.7)$ & 0.190 \\
\hline Dizziness & $38(7.9)$ & $16(5.7)^{\mathrm{a}}$ & $9(6.8)^{\mathrm{a}}$ & $13(18.6)^{b}$ & 0.001 \\
\hline Diarrhea & $17(3.5)$ & $10(3.6)$ & $7(5.3)$ & $0(0)$ & 0.154 \\
\hline Nausea and vomiting & $32(6.6)$ & $18(6.4)$ & $9(6.8)$ & $5(7.1)$ & 0.974 \\
\hline Myalgia & $93(19.3)$ & $45(16.1)^{\mathrm{a}}$ & $11(8.3)^{\mathrm{a}}$ & $37(52.9)^{\mathrm{b}}$ & $<0.001$ \\
\hline Inability to taste and smell & $5(1)$ & $4(1.4)$ & $1(0.8)$ & $0(0)$ & 0.583 \\
\hline
\end{tabular}

Data are presented as mean $\pm \mathrm{SD}$ or $n(\%)$. ANOVA, Pearson chi-square test. Different lowercase letters in a row indicate statistically significant difference between groups 
The length of stay in the intensive care unit of the patients between the ages of 75-84 was higher than the other two groups $(p=0.013)$.

Mortality rates were lowest in patients aged $65-74$ years $(p<0.001)$.

It was determined that the mortality development time of the patients aged 85 years and over was lower than the other two age groups $(p=0.040)$ (Table 2).

The mean age of patients hospitalized in intensive care was higher than those who were not hospitalized $(p<0.001)$. There was no difference in terms of gender distribution according to intensive care admission $(p=0.919)$. There was no significant difference in the incidence of HT ( $p=0.147)$, DM $(p=0.082)$, cardiovascular disease $(p=0.391)$, chronic lung disease $(p=0.999)$, heart failure $(p=0.084)$, and hyperlipidemia $(p=0.741)$.

While the frequency of COPD-bronchial asthma comorbidity was higher in patients not admitted to intensive care $(p=$ $0.002)$, malignancy $(p=0.004)$, cerebrovascular disease $(p<0.001)$, and chronic renal failure $(p<0.001)$ were higher in patients hospitalized in intensive care.

While the rate of weakness ( $p=0.006)$, dry cough $(p=0.001)$, and sore throat symptoms was higher in patients not admitted to intensive care, shortness of breath $(p<0.001)$ and dizziness $(p=$ 0.038 ) were higher in patients admitted to intensive care.

According to ICU admission, there were no differences in terms of rates of fever $(p=0.331)$, chest pain $(p=0.772)$, headache $(p=0.486)$, diarrhea $(p=0.560)$, nausea-vomiting $(p=$ $0.855)$, myalgia $(p=0.877)$, and odor-taste loss $(p=0.595)$.

The rate of ground glass opacity on tomography was higher in patients hospitalized in intensive care $(p<0.001)$.

There was no significant difference in terms of positivity of the 1st, 2nd, and 3rd PCR tests according to the hospitalization of the patients in intensive care ( $p=0.578, p=0.492, p=$ 0.999, respectively) (Table 3).
The mean age of the patients who died was higher than those without $(p<0.001)$. There was no difference in gender distribution according to mortality $(p=0.734)$.

There was no significant difference in terms of incidence rates of hypertension $(p=0.692), \mathrm{DM}(p=0.427)$, cardiovascular disease $(p=0.583)$, chronic lung disease $(p=0.504)$, heart failure $(p=0.379)$, and hyperlipidemia $(p=0.700)$ in patients with mortality.

Although the percentage of chronic renal failure was higher in patients who died, this difference was not statistically significant $(p=0.051)$.

While the rate of COPD-bronchial asthma was higher in surviving patients $(p=0.001)$, malignancy $(p=0.005)$ and cerebrovascular disease $(p<0.001)$ were higher in patients who died.

While the rate of fatigue, dry cough, and sore throat symptoms was higher in surviving patients $(p<0.001)$, the rate of shortness of breath was higher in patients who died ( $p=$ $0.005)$.

In patients who died, no difference was observed in terms of fever $(p=0.886)$, chest pain $(p=0.538)$, headache $(p=$ $0.236)$, dizziness $(p=0.101)$, diarrhea $(p=0.999)$, nauseavomiting $(p=0.858)$ myalgia $(p=0.267)$, and odor-inability to taste $(p=0.595)$ (Table 4$)$.

In patients with mortality, values of glucose $(p=0.027)$, BUN $(p<0.001)$, creatinine $(p=0.039)$, AST $(p=0.003)$, total bilirubin $(p=0.002)$, direct bilirubin $(p<0.001), \mathrm{CRP}$ $(p<0.001)$, WBC $(p<0.001)$, neutrophil $(p<0.001)$, monocyte $(p=0.024)$, troponin $\mathrm{T}(p<0.001)$, prothrombin time $(p<0.001)$, INR $(p<0.001), \mathrm{D}$-dimer $(p<0.001)$, ferritin $(p<0.001)$, and procalcitonin $(p<0.001)$ were higher.

There was no significant difference in terms of sodium $(p=0.194)$, potassium $(p=0.890), \operatorname{ALT}(p=0.453), \mathrm{CK}$ $(p=0.236)$, PLT $(p=0.371)$, lymphocyte $(p=0.148)$, APTT $(p=0.375)$, and sedimentation values $(p=0.084)$.

Table 2 Mechanical ventilation need and prognosis of patients

\begin{tabular}{|c|c|c|c|c|c|}
\hline & Total $(n: 483)$ & $65-74(n: 280)$ & $75-84(n: 133)$ & 85 and over $(n: 70)$ & $p$ \\
\hline Non-invasive mechanical ventilation & $49(10.1)$ & $25(8.9)$ & $20(15)$ & $4(5.7)$ & 0.065 \\
\hline Invasive mechanical ventilation & $71(14.7)$ & $24(8.6)^{\mathrm{a}}$ & $29(21.8)^{\mathrm{b}}$ & $18(25.7)^{\mathrm{b}}$ & $<0.001$ \\
\hline \multicolumn{6}{|l|}{ Prognosis } \\
\hline Discharge from the emergency room & $216(44.7)$ & $152(54.3)^{\mathrm{a}}$ & $43(32.3)^{b}$ & $21(30)^{\mathrm{b}}$ & \multirow[t]{3}{*}{$<0.001$} \\
\hline Pandemic clinic & $155(32.1)$ & $84(30)^{\mathrm{a}}$ & $50(37.6)^{\mathrm{a}}$ & $21(30)^{\mathrm{a}}$ & \\
\hline Intensive care unit & $112(23.2)$ & $44(15.7)^{\mathrm{a}}$ & $40(30.1)^{\mathrm{b}}$ & $28(40)^{\mathrm{b}}$ & \\
\hline Transfer to the service and intensive care & $57(27.1)$ & $22(20.8)$ & $24(33.3)$ & $11(34.4)$ & 0.109 \\
\hline Duration of stay (days) & & $5(1-21)$ & $5(1-26)$ & $4(1-13)$ & 0.275 \\
\hline Intensive care stay (days) & $5(1-75)$ & $3(1-46)^{\mathrm{a}}$ & $8(1-75)^{\mathrm{b}}$ & $3(1-30)^{\mathrm{a}}$ & 0.013 \\
\hline Mortality & $81(16.8)$ & $29(10.4)^{\mathrm{a}}$ & $34(25.6)^{\mathrm{b}}$ & $18(25.7)^{\mathrm{b}}$ & $<0.001$ \\
\hline Mortality time (days) & $11(1-75)$ & $12(1-53)^{\mathrm{a}}$ & $13(1-75)^{\mathrm{a}}$ & $6(1-38)^{b}$ & 0.040 \\
\hline
\end{tabular}

Data are presented as median (min-max) or $n(\%)$. Kruskal-Wallis test, Pearson chi-square test. Different lowercase letters in a row indicate statistically significant difference between groups 
Table 3 Characteristics of comorbidity and symptom in patients transferred from service to intensive care

\begin{tabular}{|c|c|c|c|}
\hline & Pandemic clinic $(n: 371)$ & Intensive care unit ( $n: 112)$ & $p$ \\
\hline Age & $73.51 \pm 7.51$ & $77.52 \pm 8.19$ & $<0.001$ \\
\hline \multicolumn{4}{|l|}{ Gender } \\
\hline Female & $157(42.3)$ & $48(42.9)$ & \multirow[t]{2}{*}{0.919} \\
\hline Male & $214(57.7)$ & $64(57.1)$ & \\
\hline \multicolumn{4}{|l|}{ The comorbidities } \\
\hline Hypertension & $248(66.8)$ & $83(74.1)$ & 0.147 \\
\hline Diabetes mellitus & $107(28.8)$ & $42(37.5)$ & 0.082 \\
\hline COPD-asthma-bronchitis & $157(42.3)$ & $29(25.9)$ & 0.002 \\
\hline Malignancy & $48(12.9)$ & $27(24.1)$ & 0.004 \\
\hline Cardiovascular disease & $139(37.5)$ & $47(42)$ & 0.391 \\
\hline Cerebrovascular disease & $53(14.3)$ & $38(33.9)$ & $<0.001$ \\
\hline Chronic renal failure & $23(6.2)$ & $21(18.8)$ & $<0.001$ \\
\hline Chronic liver disease & $13(3.5)$ & $4(3.6)$ & 0.999 \\
\hline Heart failure & $43(11.6)$ & $20(17.9)$ & 0.084 \\
\hline Hyperlipidemia & $10(2.7)$ & $2(1.8)$ & 0.741 \\
\hline \multicolumn{4}{|l|}{ The symptoms } \\
\hline Fever & $176(47.4)$ & $59(52.7)$ & 0.331 \\
\hline Weakness & $191(51.5)$ & $41(36.6)$ & 0.006 \\
\hline Dry cough & $195(52.6)$ & $39(34.8)$ & 0.001 \\
\hline Throat ache & $160(43.1)$ & $29(25.9)$ & 0.001 \\
\hline Shortness of breath & $197(53.2)$ & $81(72.3)$ & $<0.001$ \\
\hline Chest pain & $40(10.8)$ & $11(9.8)$ & 0.772 \\
\hline Headache & $49(13.2)$ & $12(10.7)$ & 0.486 \\
\hline Dizziness & $24(6.5)$ & $14(12.5)$ & 0.038 \\
\hline Diarrhea & $12(3.2)$ & $5(4.5)$ & 0.560 \\
\hline Nausea and vomiting & $25(6.7)$ & $7(6.3)$ & 0.855 \\
\hline Myalgia & $72(19.4)$ & $21(18.8)$ & 0.877 \\
\hline Smell-inability to taste & $5(1.3)$ & $0(0)$ & 0.595 \\
\hline Ground glass opacity & $157(45.5)$ & $88(81.5)$ & $<0.001$ \\
\hline \multicolumn{4}{|l|}{ PCR test result } \\
\hline Negative & $357(96.5)$ & $106(95.5)$ & \multirow[t]{2}{*}{0.578} \\
\hline Positive & $13(3.5)$ & $5(4.5)$ & \\
\hline \multicolumn{4}{|l|}{ 2.PCR test result } \\
\hline Negative & $138(95.8)$ & $58(93.5)$ & \multirow[t]{2}{*}{0.492} \\
\hline Positive & $6(4.2)$ & $4(6.5)$ & \\
\hline \multicolumn{4}{|l|}{ 3.PCR test result } \\
\hline Negative & $24(92.3)$ & $14(93.3)$ & \multirow[t]{2}{*}{0.999} \\
\hline Positive & $2(7.7)$ & $1(6.7)$ & \\
\hline
\end{tabular}

Data are presented as mean $\pm \mathrm{SD}$ or $n(\%)$. Student's $t$ test, Pearson chi-square test, Fisher's exact test
The rate of ground glass opacity in thorax CT was higher in patients with mortality $(p<0.001)$. There was no significant difference in terms of positivity of the 1st, 2nd, and 3rd PCR tests of the patients with mortality $(p=0.515, p=0.999, p=$ 0.999 , respectively).

In patients who were positive in the first PCR test and negative in subsequent tests, there was no difference in the duration to yield negative according to mortality $(p=0.200)$. Likewise, the duration of the patients who became positive when the first PCR test was negative did not differ according to mortality $(p=0.333)$.

The rate of using non-invasive mechanical ventilation and invasive mechanical ventilation was higher in patients with mortality $(p<0.001)$.

While the group of patients discharged from the emergency department and hospitalized in the service had the highest surveillance, mortality was significantly higher in those who were admitted to the intensive care unit $(p<0.001)$. 
Table 4 Characteristics of comorbidity and symptom in patients with mortality

\begin{tabular}{|c|c|c|c|}
\hline & Discharged $(n: 280)$ & Died $(n: 133)$ & $p$ \\
\hline Age & $73.75 \pm 7.66$ & $77.86 \pm 7.93$ & $<0.001$ \\
\hline \multicolumn{4}{|l|}{ Gender } \\
\hline Female & $172(42.8)$ & $33(40.7)$ & \multirow[t]{2}{*}{0.734} \\
\hline Male & $230(57.2)$ & $48(59.3)$ & \\
\hline \multicolumn{4}{|l|}{ The comorbidities } \\
\hline Hypertension & $277(68.9)$ & $54(66.7)$ & 0.692 \\
\hline Diabetes mellitus & $121(30.1)$ & $28(34.6)$ & 0.427 \\
\hline COPD-asthma-bronchitis & $168(41.8)$ & $18(22.2)$ & 0.001 \\
\hline Malignancy & $54(13.4)$ & $21(25.9)$ & 0.005 \\
\hline Cardiovascular disease & $157(39.1)$ & $29(35.8)$ & 0.583 \\
\hline Cerebrovascular disease & $62(15.4)$ & $29(35.8)$ & $<0.001$ \\
\hline Chronic renal failure & $32(8)$ & $12(14.8)$ & 0.051 \\
\hline Chronic liver disease & $13(3.2)$ & $4(4.9)$ & 0.504 \\
\hline Heart failure & $50(12.4)$ & $13(16)$ & 0.379 \\
\hline Hyperlipidemia & $11(2.7)$ & $1(1.2)$ & 0.700 \\
\hline \multicolumn{4}{|l|}{ The symptoms } \\
\hline Fever & $195(48.5)$ & $40(49.4)$ & 0.886 \\
\hline Weakness & $208(51.7)$ & $24(29.6)$ & $<0.001$ \\
\hline Dry cough & $212(52.7)$ & $22(27.2)$ & $<0.001$ \\
\hline Throat ache & $175(43.5)$ & $14(17.3)$ & $<0.001$ \\
\hline Shortness of breath & $220(54.9)$ & $58(71.6)$ & 0.005 \\
\hline Chest pain & $44(10.9)$ & $7(8.6)$ & 0.538 \\
\hline Headache & $54(13.4)$ & $7(8.6)$ & 0.236 \\
\hline Dizziness & $28(7)$ & $10(12.3)$ & 0.101 \\
\hline Diarrhea & $14(3.5)$ & $3(3.7)$ & 0.999 \\
\hline Nausea and vomiting & $27(6.7)$ & $5(6.2)$ & 0.858 \\
\hline Myalgia & $81(20.1)$ & $12(14.8)$ & 0.267 \\
\hline Inability to smell and taste & $5(1.2)$ & $0(0)$ & 0.595 \\
\hline
\end{tabular}

Data are presented as mean $\pm \mathrm{SD}$ or $n(\%)$. Student's $t$ test, Pearson chi-square test, Fisher's exact test
Patients transferred from the service to the intensive care unit had a higher mortality $(p<0.001)$. No significant difference was observed in terms of the length of stay of the patients hospitalized in the ward according to mortality $(p=0.592)$. Patients who died had higher length of stay in the intensive care unit $(p=0.001)$ (Table 5).

In multivariate logistic regression model, age, COPDbronchial asthma, weakness, dry cough, ground glass opacity, and CRP were identified as an independent predictive factor associated with mortality. Patients aged between 75 and 84 (OR: 2.602 ; 95\% CI: $1.306-5.183 ; p=0.007$ ) or $\geq 85$ (OR: 4.086 ; 95\% CI: $1.687-9.9 ; p=0.002$ ) had higher risk for mortality compared to patients aged between 65 and 74 . Patients with COPD (OR: 0.377; 95\% CI: 0.187-0.762; $p=0.007$ ), weakness (OR: $0.415 ; 95 \%$ CI: $0.197-0.874 ; p=0.021)$, and dry cough (OR: $0.479 ; 95 \%$ CI: $0.23-0.994 ; p=0.048)$ had lower risk for mortality. Patients with ground glass opacity were 2.54 times higher risk for mortality than patients with normal CT (OR: 2.539; 95\% CI: $1.163-5.543 ; p=0.019$ ).
Increasing CRP (C-reactive protein) (OR: $1.01 ; 95 \% \mathrm{CI}$ : $1.006-1.013 ; p<0.001)$ was positively associated with mortality (Table 6) (Fig. 7).

It was seen that the rate of ground glass opacity of patients who died aged 65-74 and 75-84 years was statistically higher than those who survived ( $p<0.001, p=0.001$, respectively). Although it was observed that the rate of ground glass opacity in patients aged 85 years and older was higher than those who survived, this difference was not statistically significant $(p=$ 0.079) (Fig. 1).

No significant difference was found in terms of PCR test positivity rates according to mortality in patients aged $65-74$, between 75 and 84 years, and over 85 years $(p=0.627, p=$ $0.161, p=0.999$, respectively) (Fig. 2).

It was seen that the rate of ground glass opacity of the patients between the ages of 65-74, between the ages of 7584 , and those who were hospitalized in intensive care at the age of 85 and above was statistically higher than those without ( $p<0.001, p<0.001, p=0.045$, respectively) (Fig. 3). 
Table 5 Laboratory findings of patients with mortality

\begin{tabular}{|c|c|c|c|}
\hline & Survived ( $n: 402)$ & Died $(n: 81)$ & $p$ \\
\hline Glucose & $123.5(11-1065)$ & $149(32-636)$ & 0.027 \\
\hline BUN & $20(2.7-222)$ & $29(9-175)$ & $<0.001$ \\
\hline Creatinine & $1(0.5-7.24)$ & $1.1(0.5-21)$ & 0.039 \\
\hline $\mathrm{Na}$ & $136(113-157)$ & $136(122-180)$ & 0.194 \\
\hline K & $4(2.6-44)$ & $4(2.7-6.3)$ & 0.890 \\
\hline Calcium & $9(5.6-96)$ & $8.3(6.7-12.5)$ & $<0.001$ \\
\hline ALT & $17(3-1129)$ & $17(4-1279)$ & 0.453 \\
\hline AST & $22(8-1152)$ & $28(8-2654)$ & 0.003 \\
\hline Creatine kinase & $78(7-6590)$ & $96(6-2195)$ & 0.236 \\
\hline Total bilirubin & $0.58(0.14-6.59)$ & $0.71(0.16-31.78)$ & 0.002 \\
\hline Direct bilirubin & $0.11(0.01-4.15)$ & $0.2(0.04-15.94)$ & $<0.001$ \\
\hline CRP & $20.7(0.09-317.5)$ & $75.3(0.22-388.3)$ & $<0.001$ \\
\hline WBC & $10(0.1-32.5)$ & $12.2(1.3-103)$ & $<0.001$ \\
\hline HGB & $12.2 \pm 2.32$ & $11.17 \pm 2.3$ & 0.001 \\
\hline HTC & $37.1(12-322)$ & $34.9(16.8-52.1)$ & 0.001 \\
\hline PLT & $236(4-884)$ & $271(20-664)$ & 0.371 \\
\hline NEU \# & $7.2(0.1-83.5)$ & $9.5(0.2-90)$ & $<0.001$ \\
\hline LYM \# & $1.35(0.1-22.9)$ & $1.1(0.3-37.9)$ & 0.148 \\
\hline MON \# & $0.7(0-10.2)$ & $0.9(0.01-43.1)$ & 0.024 \\
\hline Troponin $\mathrm{T}$ & $19(3-6350)$ & $47(3-1402)$ & $<0.001$ \\
\hline APTT & $30.7(16.6-120.9)$ & $30.7(22-101.9)$ & 0.375 \\
\hline Prothrombin time & $12.7(9-45.5)$ & $13.8(10.5-97.1)$ & $<0.001$ \\
\hline INR & $1.09(0.79-4.02)$ & $1.23(0.91-8.13)$ & $<0.001$ \\
\hline D-dimer & $358(46.3-11,826)$ & $712(51-25,403)$ & $<0.001$ \\
\hline Sedimentation & $28(2-110)$ & $44(4-140)$ & 0.084 \\
\hline Ferritin & $96(6.1-2656)$ & $320(11-3189)$ & $<0.001$ \\
\hline Procalcitonin & $0.11(0.01-666.2)$ & $0.51(0.03-61)$ & $<0.001$ \\
\hline Ground glass opacity & $179(47.9)$ & $66(83.5)$ & $<0.001$ \\
\hline \multicolumn{4}{|l|}{ PCR Test result } \\
\hline Negative & $388(96.5)$ & $75(94.9)$ & \multirow[t]{2}{*}{0.515} \\
\hline Positive & $14(3.5)$ & $4(5.1)$ & \\
\hline \multicolumn{4}{|l|}{ 2.PCR test result } \\
\hline Negative & $154(95.1)$ & $42(95.5)$ & \multirow[t]{2}{*}{0.999} \\
\hline Positive & $8(4.9)$ & $2(4.5)$ & \\
\hline \multicolumn{4}{|l|}{ 3.PCR test result } \\
\hline Negative & $28(93.3)$ & $10(90.9)$ & \multirow[t]{2}{*}{0.999} \\
\hline Positive & $2(6.7)$ & $1(9.1)$ & \\
\hline If the first PCR test is positive, after how many days it becomes negative & $6(3-16)$ & $23(23-23)$ & 0.200 \\
\hline If the first PCR is negative, the blood became pcr + days later & $1(1-2)$ & $15(15-15)$ & 0.333 \\
\hline Non-invasive mechanical ventilation & $22(5.5)$ & $27(33.3)$ & $<0.001$ \\
\hline Invasive mechanical ventilation & $7(1.7)$ & $64(79)$ & $<0.001$ \\
\hline \multicolumn{4}{|l|}{ Prognosis } \\
\hline Discharge from the emergency room & $213(53)^{\mathrm{a}}$ & $3(3.7)^{\mathrm{b}}$ & \multirow[t]{3}{*}{$<0.001$} \\
\hline Service & $145(36.1)^{\mathrm{a}}$ & $10(12.3)^{\mathrm{b}}$ & \\
\hline Intensive care & $44(10.9)^{\mathrm{a}}$ & $68(84)^{\mathrm{b}}$ & \\
\hline Is there a transfer from the service to intensive care? & $29(16.9)$ & $28(73.7)$ & $<0.001$ \\
\hline Duration of stay (days) & $5(1-21)$ & $5(1-26)$ & 0.592 \\
\hline Intensive care stay (days) & $3(1-46)$ & $8(1-75)$ & 0.001 \\
\hline
\end{tabular}

Data are presented as mean $\pm \mathrm{SD}$, median (min-max), or $n(\%)$. Student's $t$ test, Mann-Whitney $U$ test, Pearson chi-square test. Different lowercase letters in a row indicate statistically significant difference between groups 
Table 6 Factors affecting mortality

\begin{tabular}{lll}
\hline & OR $(95 \%$ CI $)$ & $p$ \\
\hline Age & & \\
$65-74$ & Reference & - \\
$75-84$ & $2.602(1.306-5.183)$ & 0.007 \\
$\geq 85$ & $4.086(1.687-9.9)$ & 0.002 \\
COPD-asthma-bronchitis & $0.377(0.187-0.762)$ & 0.007 \\
Malignancy & $1.808(0.881-3.709)$ & 0.106 \\
Cerebrovascular disease & $1.104(0.549-2.22)$ & 0.782 \\
Chronic renal failure & $1.208(0.473-3.083)$ & 0.693 \\
Weakness & $0.415(0.197-0.874)$ & 0.021 \\
Dry cough & $0.479(0.23-0.994)$ & 0.048 \\
Throat ache & $0.577(0.231-1.44)$ & 0.238 \\
Shortness of breath & $1.516(0.78-2.947)$ & 0.220 \\
Ground glass opacity & $2.539(1.163-5.543)$ & 0.019 \\
CRP & $1.01(1.006-1.013)$ & $<0.001$ \\
\hline
\end{tabular}

No significant difference was found in terms of PCR test positivity rates according to mortality in patients aged 65-74, between 75 and 84 years, and over 85 years $(p=0.999, p=$ $0.215, p=0.999$, respectively) (Fig. 4).

Using ROC analysis for mortality, the optimal cutoff point for CRP according to the Youden index was $\geq$ 60.35 (area under the curve $(\mathrm{AUC})=0.712(95 \% \mathrm{CI}$ : $0.646-0.778, p<0.001)$ ), sensitivity $57.9 \%$, and specificity $76.3 \%$ (Fig. 5).

A ROC analysis of age for mortality is given in Fig. 3. AUC value for age was 0.650 (95\% CI: 0.585-0.715, $p<0.001)$. The optimal cutoff value of age for mortality was $\leq 71.5$, and sensitivity and specificity were $76.5 \%$ and $50.2 \%$, respectively (Fig. 6).

\section{Discussion}

Having a low rate of geriatric COVID-19 diagnosis group with $13.3 \%$ (483) of 3628 COVID-19 symptom patients who applied to the emergency department of the 3rd degree regional hospital within the specified periods is an indicator of not only the effective implementation of health policies in Turkey especially regarding the geriatric population in the COVID-19 pandemic, but it also indicates how meticulously personal protective measures are applied such as maintaining social distance, isolation at home, and using surgical masks. Nevertheless, the COVID-19 pandemic is devastating for the geriatric population, with high morbidity and mortality levels [2]. Although, in the literature, for the geriatric age groups, cardiac arrest, sepsis, and cardiovascular diseases are seen as the highest mortality cause in all three groups in intensive care [9], it is clear that COVID-19 will be included in this ranking during the COVID-19 pandemic period. In the literature, there are studies reporting that the prognosis of COVID-19 pandemic is more severe in men [10]. In our study, although the mortality rate was $59.3 \%$ (48) higher in the male geriatric group as a numerical value, no significant difference between genders was observed. In the literature, the sensitivity of tomography in the diagnosis of COVID-19 was 97.2\%, while the sensitivity of rRT-PCR was only $83.3 \%$ [11]. However, it should be kept in mind that false-negative results may occur in rRT-PCR results in the geriatric patient group [12]. In our study, while the rate of transfer to intensive care and mortality results increased positively in geriatric patients with ground glass opacity on tomography, the same significant results were not obtained with the rRT-PCR results. In the light of these findings, making a diagnosis and treatment decision by only looking at rRT-PCR results and considering only rRT-PCR results in mass screening gives erroneous results and makes
Fig. 1 Ground glass opacity distribution in patients with mortality
Thoracic CT image of ground-glass opacities in the lung

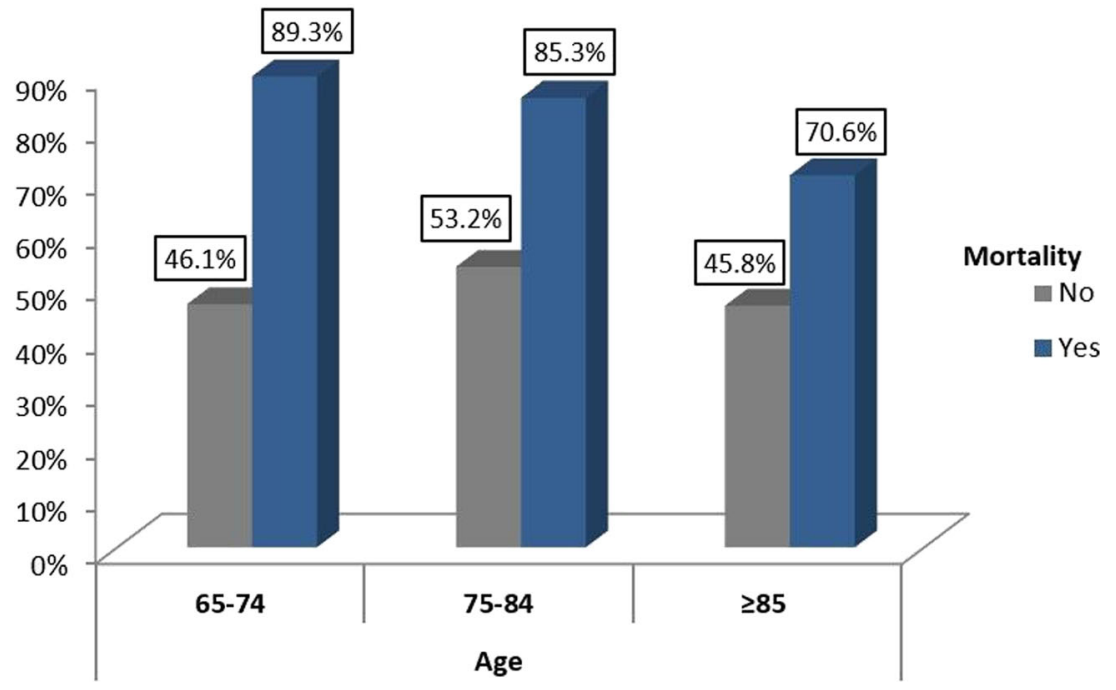


Fig. 2 PCR positivity in patients with mortality
rRT-PCR

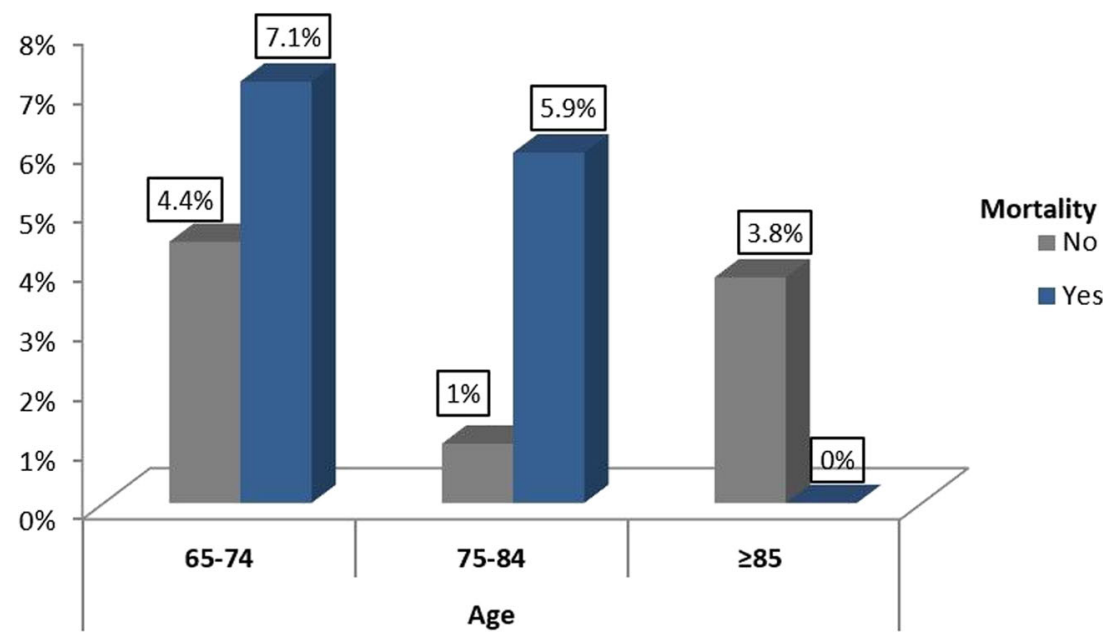

pandemic control difficult [12]. In the management of COVID-19 infection, both lung and cardiovascular complications increase significantly, especially in patients with only cardiovascular disease comorbidity [13]. In our study, there was no significant increase in mortality in patients with cardiovascular disease, and interestingly, in patients with dry cough symptoms and chronic obstructive pulmonary disease (COPD) and bronchial asthma comorbidity, ICU transfer rate and mortality appear to be low risk factors. This situation may be related to the more effective application of personal protective factors in patients with comorbidities related to the lungs, and to pneumococcal vaccination routinely applied to geriatric patients with lung disease over the age of 65 in Turkey. Studies have reported that pneumococcal and influenza vaccination reduces the mortality rate from COVID-19 in the elderly [14]. In a retrospective study in the literature, it was determined that there was a weak and positive correlation between age and mortality in geriatric patients, while the rRT-PCR test result had no effect on mortality [15]. In our study, age, COPD-bronchial asthma, malaise, dry cough, ground glass opacity, and CRP were identified as an independent predictive factor associated with mortality. In our study, the risk of mortality increases with age, and when age is $\leq$ 71.5 , it predicts mortality with $76.5 \%$ sensitivity and $50.2 \%$ specificity. In our study, no significant difference was found in terms of mortality and PCR test positivity. The rate of invasive mechanical ventilation use was lower in the 65-74 age group compared to the other two age groups, and the highest rate of patients discharged from the emergency department was between the ages of 65-74, while the rate of admission to intensive care was observed to be the highest in patients between the ages of 75-84 and 85 and older. This situation is closely related to the general condition disorder induced by COVID-19, which is caused by the increase of
Fig. 3 Ground glass opacity according to age groups in patients transferred to intensive care
Thoracic CT image of ground-glass opacities in the lung

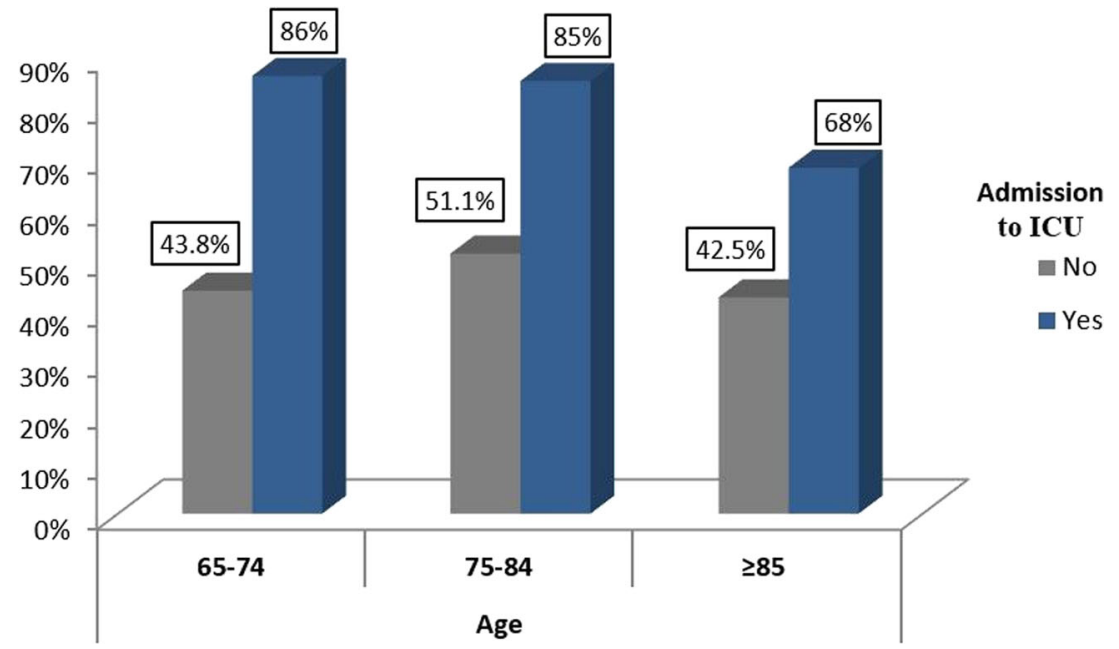


Fig. 4 PCR positivity in intensive care patients

\section{rRT-PCR}

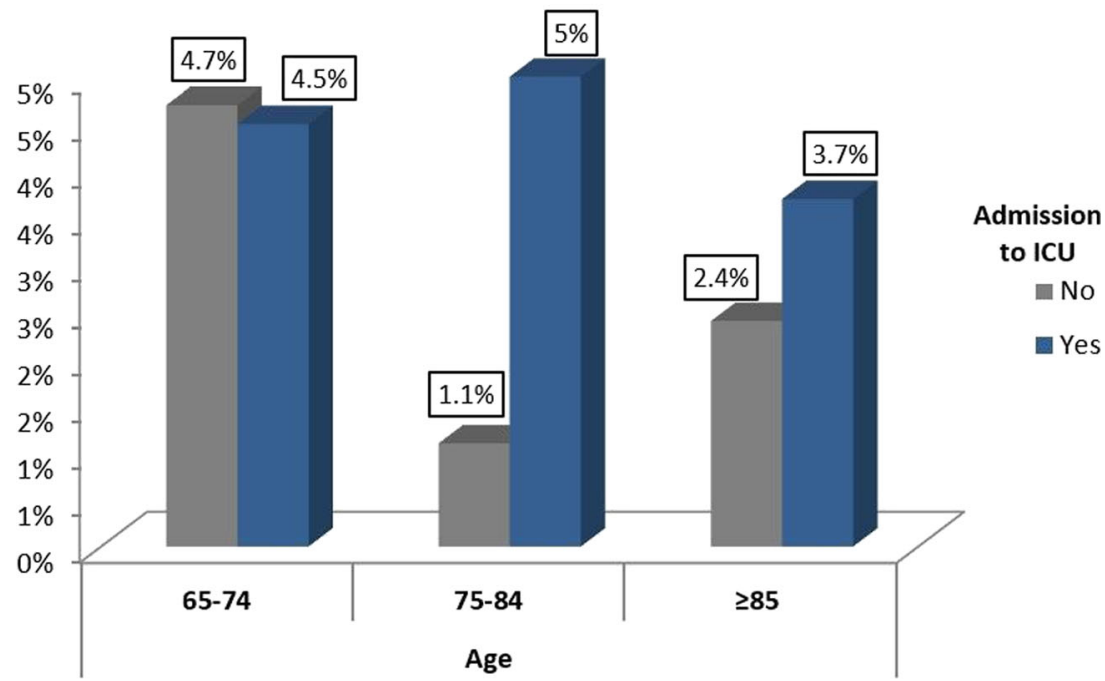

multimorbidity in advanced age. In the literature, patients with a diagnosis of COVID-19 who received a standard dose of prophylactic anticoagulant had higher D-dimer levels in their laboratory findings, while ESR, CRP, fibrinogen, ferritin, and procalcitonin were higher in patients with thrombotic complications compared to those without [16]. Our patients were treated with Oksapar 6000 ANTI-XA IU/0.6 mL for prophylactic anticoagulation. When evaluated in terms of mortality, glucose, BUN, creatinine, AST, total bilirubin, direct bilirubin, CRP, WBC, neutrophil, monocyte, troponin T, prothrombin time, INR, D-dimer, ferritin, and procalcitonin values were observed to be higher. There was no significant difference in terms of sodium, potassium, ALT, creatine kinase, platelet, lymphocyte, APTT, and sedimentation values. When CRP is $\geq 60.35$, mortality can be predicted with $57.9 \%$ sensitivity and $76.3 \%$ specificity. In a meta-analysis study, chronic obstructive pulmonary disease $(\mathrm{OR}=5.08)$ was associated with comorbidities including diabetes $(\mathrm{OR}=$ 3.17 ) in patients who needed an intensive care unit,

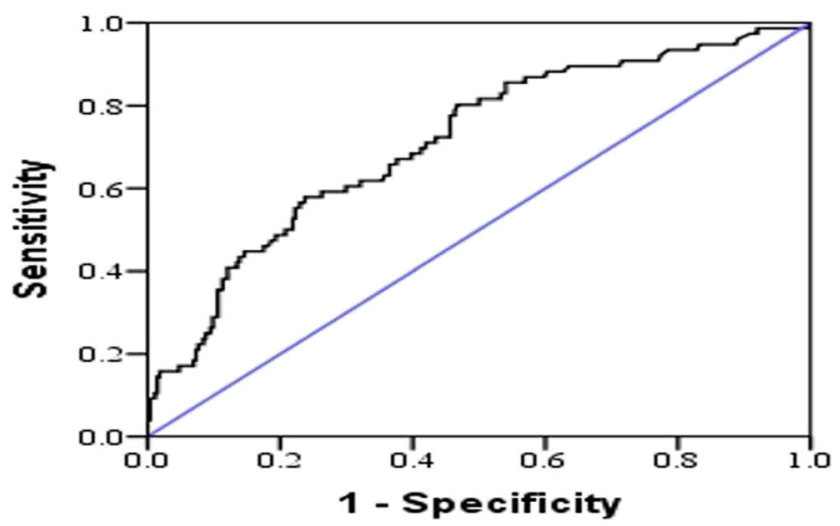

Fig. 5 ROC curve analysis for CRP to predict mortality hypertension $(\mathrm{OR}=2.40)$, coronary heart disease ( $\mathrm{OR}=$ $2.66)$, cerebrovascular diseases $(\mathrm{OR}=2.68)$ and malignancy $(\mathrm{OR}=2.21)$, dyspnea, decreased lymphocyte and platelet counts, and increased leukocyte count; the levels of Creactive protein, procalcitonin, lactate dehydrogenase, aspartate aminotransferase, alanine aminotransferase, creatine kinase and creatinine were determined to be significantly different, and it was also found that the morbidity and mortality rate increased for this patient group [17]. Although our study displays a low rate in the geriatric population $(13.3 \%, 483)$ due to COVID-19, the COVID-19 pandemic continues to be a threat in geriatric age groups. We are of the idea that this rate will decrease even more with the spread of personal protective measures applied in geriatric age groups and close follow-up of the elderly with comorbidities in Turkey and all over the world (Fig. 7).

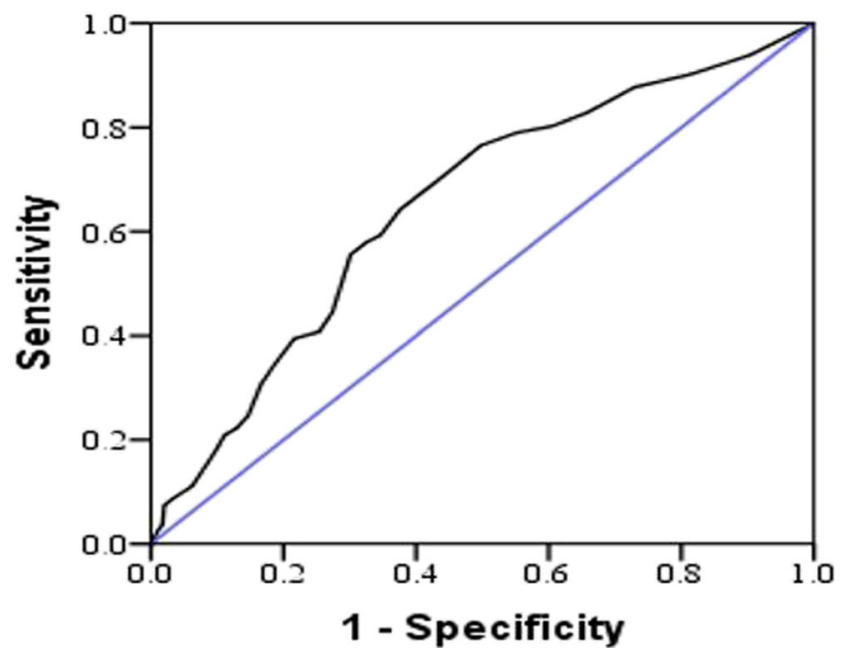

Fig. 6 ROC curve analysis for age to predict mortality 


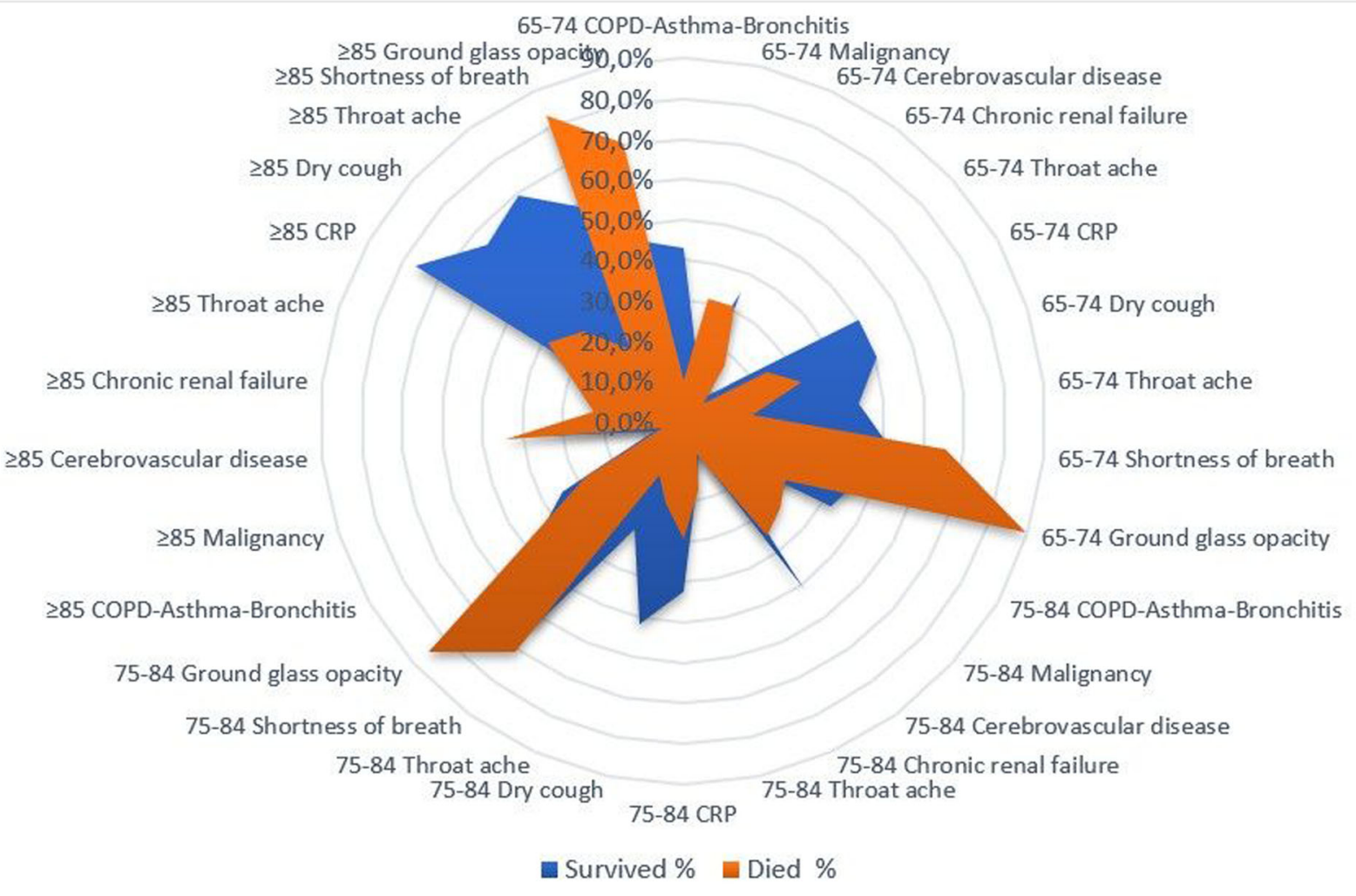

Fig. 7 Factors affecting mortality

\section{Conclusion}

Mortality rates were lowest in patients aged 65-74 years. While PCR positivity has no effect on mortality in 3 different geriatric age groups, the ground glass opacity in tomography is closely related to the need for intensive care and increased mortality. Mortality rate is high in geriatric patients who need mechanical ventilation in intensive care. In patients with COPD-bronchial asthma comorbidity and those with symptoms of fatigue, dry cough, and sore throat, transfer to intensive care and mortality rates were lower, while patients who were transferred to intensive care and developed mortality had higher malignancy and cerebrovascular disease comorbidities and dyspnea symptoms. Advanced age, dyspnea symptom, ground glass opacity in lung computerized tomography, and high CRP are among the indicators of risk factors that increase mortality in patients with COVID-19. When age is $\leq 71.5$, mortality is predicted with $76.5 \%$ sensitivity and $50.2 \%$ specificity, and when CRP is $\geq 60.35$, mortality is predicted with $57.9 \%$ sensitivity and $76.3 \%$ specificity.

\section{Code Availability Available.}

Authors' Contributions OG: Manuscript writing/editing.

MD: Data collection or management.

\section{Compliance with Ethical Standards}

Conflict of Interest The authors declare that they have no conflict of interest.

Ethical Approval This study was conducted in accordance with the Declaration of Helsinki from obtaining approval from the Institutional Ethics Committee (Date of Approval-Protocol No: 03.06.2020-7/8).

\section{References}

1. Mayr V, Streit BN, Gartlehner G. Quarantine alone or in combination with other public health measures to control COVID-19: a rapid review (review). Gesundheitswesen. 2020;82(6):501-6.

2. Farrell TW, Francis L, Brown T, Ferrante LE, Widera E, Rhodes R, et al. Rationing limited health care resources in the COVID-19 era and beyond: ethical considerations regarding older adults. J Am Geriatr Soc. 2020;68(6):1143-9.

3. Desideri I, Pilleron S, Battisti NML et al. Caring for older patients with cancer during the COVID-19 pandemic: a young International Society of Geriatric Oncology (SIOG) global perspective. J Geriatr Oncol. 2020;11(7):1175-81.

4. Sharma A, Crosby DL. Special considerations for elderly patients with head and neck cancer during the COVID-19 pandemic. Head Neck. 2020;42(6):1147-9.

5. Newport KB, Malhotra S, Widera E. Prognostication and proactive planning in COVID-19. J Pain Symptom Manag. 2020;60(2):e525.

6. Bonanad C, García-Blas S, Tarazona-Santabalbina FJ et al. [Coronavirus: the geriatric emergency of 2020. Joint document of 
the Geriatric Cardiology Section of the Spanish Society of Cardiology and the Spanish Society of Geriatrics and Gerontology]. Rev Esp Cardiol. 2020;73(7):569-76.

7. Kara M, Ekiz T, Ricci V, et al. 'Scientific strabismus' or two related pandemics: COVID-19 \& vitamin D deficiency. Br J Nutr. 2020;12:1-20.

8. Chakraborty C, Sharma AR, Sharma G, Bhattacharya M, Lee SS. SARS-CoV-2 causing pneumonia-associated respiratory disorder (COVID-19): diagnostic and proposed therapeutic options. Eur Rev Med Pharmacol Sci. 2020;24(7):4016-26.

9. Görgülü Ö, Koșar MN. Diagnosis and mortality outcomes of three different geriatric age groups in the intensive care unit: 10-year retrospective analysis. Turk J Geriatr. 2020;23(1):66-74.

10. Haitao T, Vermunt JV, Abeykoon J, Ghamrawi R, Gunaratne M, Jayachandran M, et al. COVID-19 and sex differences: mechanisms and biomarkers. Mayo Clin Proc. 2020;95(10):2189-203.

11. Long C, Xu H, Shen Q, Zhang X, Fan B, Wang C, et al. Diagnosis of the coronavirus disease (COVID-19): rRT-PCR or CT? Eur J Radiol. 2020;126:108961.

12. Görgülü Ö, Duyan M. rRT-PCR results of a Covid-19 diagnosed geriatric patient. SN Comprehensive Clinical Medicine. SN Compr Clin Med. 2020;1-4.
13. Li G, Hu R, Gu X. A close-up on COVID-19 and cardiovascular diseases. Nutr Metab Cardiovasc Dis. 2020;30(7):1057-60.

14. Thindwa D, Quesada MG, Liu Y, et al. Use of seasonal influenza and pneumococcal polysaccharide vaccines in older adults to reduce COVID-19 mortality. Vaccine. 2020;38(34):5398-401.

15. Smet RD, Mellaerts B, Vandewinckele H, et al. Frailty and mortality in hospitalized older adults with COVID-19: retrospective observational study. J Am Med Dir Assoc. 2020;21(7):928-932.e1.

16. Samkari HA, Leaf RSK, Dzik WH, et al. COVID-19 and coagulation: bleeding and thrombotic manifestations of SARS-CoV-2 infection. Blood. 2020;136(4):489-500.

17. Li J, He X, Yuan Y, et al. Meta-analysis investigating the relationship between clinical features, outcomes, and severity of severe acute respiratory syndrome coronavirus 2 (SARS-CoV-2) pneumonia. Am J Infect Control. 2020;S0196-6553(20):30369-2.

Publisher's Note Springer Nature remains neutral with regard to jurisdictional claims in published maps and institutional affiliations. 